\title{
RELACIÓN ENTRE EL ÍNDICE CPOD Y LA LIMITACIÓN AL SONREÍR EN ESCOLARES DE 12 AÑOS DE LA PARROQUIA SAN SEBASTIÁN, CUENCA-ECUADOR 2016
}

\section{RELATIONSHIP BETWEEN THE CPOD INDEX AND THE LIMITATION TO SMILE IN 12 YEARS OLD IN THE PARISH OF SAN SEBASTIAN, CUENCA-ECUADOR 2016}

\author{
Duy Zaruma Diana Elizabeth ${ }^{1}$, Patricio Fernando Sarmiento Criollo² ${ }^{2}$ Mejía Caldas Hernán Santiago ${ }^{3}$
}

1 Estudiante, Universidad Católica de Cuenca. Unidad Académica de Salud y Bienestar. Carrera de Odontología. Cuenca Ecuador.diana20duy@gmail.com

2 Catedrático, Universidad Católica de Cuenca. Unidad Académica de Salud y Bienestar. Carrera de Odontología. Cuenca-Ecuador.psarmiento@ucacue.edu.ec

3 Estudiante, Universidad Católica de Cuenca. Unidad Académica de Salud y Bienestar. Carrera de Odontología. Cuenca-Ecuador. santiago1996mc@gmail.com

https://doi.org/10.53591/eoug.v4i2.356

\author{
Correspondencia: \\ diana20duy@gmail.com \\ Recibido: 28/06/2021 \\ Aceptado: $23 / 07 / 2021$ \\ Publicado: 28/07/2021
}

\section{RESUMEN}

Objetivo: Determinar la posible relación entre el índice CPOD y la limitación al sonreír en escolares de 12 años de la parroquia San Sebastián, Cuenca-Ecuador, 2016.. Metodología: Este es un estudio descriptivo transversal en el cual se utilizaron 281 fichas pertenecientes al estudio del mapa epidemiológico de escolares de la parroquia San Sebastián, debido que todas cumplían con los requisitos de inclusión no fue necesario ningún método de descarte, se utilizaron como variables el índice CPOD para determinar la frecuencia de caries, mientras que para determinar la severidad de la limitación al sonreír se usó el índice Child-OIDP luego se procedió a relacionar las variables mediante la prueba estadística de Kendall Resultados: Los resultados obtenidos de esta investigación demostraron que el nivel de índice CPOD “ Muy bajo” es el rango predominante en esta población con un 38,1\%, también se observó que el $74,7 \%$ de los escolares no presentaron impacto al sonreír, por lo tanto el apartado "sin limitación" fue el más sobresaliente en el sexo femenino con un 75,8\% en comparación al valor de 74,5\% en el sexo masculino. Conclusiones: Sí existe una relación entre el índice CPOD y la limitación al sonreír en escolares de 12 años en la parroquia San Sebastián, Cuenca Ecuador, debido que a menor índice CPOD menor limitación al sonreír.

Palabras clave: Sonreír, calidad de vida, CPOD, Child-OIDP, escolares.

\section{ABSTRACT}

Objective: To determine the possible relationship between the CPOD index and the limitation of smiling in 12-year-old schoolchildren from the parish of San Sebastián, Cuenca-Ecuador, 2016. Methodology: This is a cross-sectional descriptive study in which 281 cards belonging to the study of the epidemiological map of schoolchildren of the San Sebastian parish were used, because all met the inclusion requirements no method of discarding was necessary, the CPOD index was used as variables to determine the frequency of caries, while the Child-OIDP index was used to determine the severity of the limitation of smiling, then the variables were related using Kendall's statistical test. Results: The results obtained from this research showed that the CPOD index level "Very low" is the predominant range in this population with $38.1 \%$, it was also observed that $74.7 \%$ of the schoolchildren did not present impact when smiling, therefore the "no limitation" section was the most outstanding in the female sex with $75.8 \%$ compared to the value of $74.5 \%$ in the male sex. Conclusions: There is a relationship between the CPOD index and the limitation of smiling in 12-year-old schoolchildren in the parish of San Sebastian, Cuenca, Ecuador, because the lower the CPOD index, the lower the limitation of smiling. Key words: Smiling, quality of life, CPOD, Child-OIDP, schoolchildren. 


\section{REVISTA CIENTÍFICA “ESPECIALIDADES ODONTOLÓGICAS UG”. ISSN: 2600576X ÓRGANO OFICIAL DE LA FACULTAD PILOTO DE ODONTOLOGÍA DE LA UNIVERSIDAD DE GUAYAQUIL}

\section{INTRODUCCIÓN}

La sonrisa es una de las expresiones más importantes del rostro ya que representa una vía de comunicación no verbal fundamental, teniendo un rol relevante en la expresión y apariencia facial 1,2 , entre las funciones principales de la sonrisa es comunicar, revelar, expresar y exhibir diferentes emociones, tales como la alegría. ${ }^{3}$ Ésta puede verse afectada debido a múltiples causas, entre ellas la caries dental, traumatismos dentoalveolares, mal posiciones dentarias, ausencia de piezas etc.

La caries dental es una enfermedad infecciosa con especies bacterianas específicas que afectan a los tejidos de soporte del diente llevándolo al deterioro y si no es tratada a tiempo la perdida en su totalidad del mismo, Esta ausencia de la pieza dentaria presenta molestias como sensibilidad, dolor y limitación funcional, y esto puede verse reflejado en las expresiones y sensaciones de incomodidad y falta de confianza a la hora de expresar un gesto, específicamente al momento de sonreír. 4,5

Muchos autores e instituciones tienen un solo propósito que es dar a conocer, prevenir y concientizar sobre los múltiples problemas tanto patológicos, funcionales y biopsicosociales que pueden causar el extravío del órgano dentario en tempranas edades, ${ }^{6}$ por consiguiente, la estética dental es un factor que se ve reflejado en el día a día del individuo, y este puede ser desfavorable cuando existe una condición que lo afecte, especialmente los adolescentes puesto que corren el riesgo de desarrollar baja autoestima generando un impacto negativo en la calidad de vida de este vulnerable grupo poblacional. 7

La calidad de vida se define como la percepción que tienen las personas en torno a su realidad, por lo tanto la salud oral incide en la calidad de vida de las personas con profundas repercusiones en el estado anímico a tal punto que quienes la padecen se limitan a sonreír ${ }^{8,9}$, es por esto que si la salud oral se ve afectada puede causar secuelas importantes en la percepción de una persona sobre sí misma, especialmente en edades tempranas.10,11 Estudios han demostrado correlación entre la prevalencia de caries y el estatus social, por lo que demuestra que la caries dental no solo puede ser eliminada con conceptos de prevención, hábitos higiénicos y dietéticos, sino también involucraría que el estado otorgue a todos un nivel por igual, es decir que todos estemos en plenas condiciones para poder acceder a un nivel de vida digna. 12,13

En la actualidad, en el Ecuador la situación de salud oral está en proceso de constante cambio, sin embargo, aún hay mucho trabajo por hacer con respecto al Ministerio de Salud Pública y su limitada atención a la población en general. ${ }^{14}$ Los reportes del MSP del Ecuador reporta altos índices de prevalencia de afecciones bucodentales en niños, consolidándose de esa manera como la patología de mayor daño entre las afecciones orales. Esta patología en los niños al no ser tratada puede repercutir como infecciones orales, mala adquisición del lenguaje, aumento de peso lento, deficiencia de nutrientes y disminución de la autoestima. ${ }^{15}$ Investigadores aseguran que las caries causan limitaciones al individuo afectando ámbitos importantes como el social y emocional. 16,17 En los niños, para la medición de caries, el índice CPOD es el más común y el más utilizado, este índice establecido por Klein, Palmer y Knutson, mide las caries dentales con la finalidad de recolectar un fundamental banco de datos con respecto a la evolución de esta patología en muchos países. ${ }^{18}$ Sus iniciales significan: dientes (D) con caries (C) perdido por caries (P) y obturados (O), La suma de estas variables determinan el índice CPOD en los adultos, sin embargo, en la dentición decidua se utiliza una variación de este índice CEO-D que significa: (c) diente temporal cariado, (e) diente temporal con extracción indicada y (o) diente temporal obturado. 19,20

El índice Child-OIDP mide el rendimiento de actividades diarias como comer, hablar, lavarse los dientes, socializar, dormir, hacer tareas, estabilidad emocional, y sonreír, afectadas por condiciones orales en los últimos 3 meses en escolares de 11 y 12 años de edad.

El objetivo de este artículo de tipo descriptivo transversal es dilucidar si existe una relación entre el índice CPOD y la limitación al sonreír en escolares de 12 años de la parroquia San Sebastián, Cuenca-Ecuador, 2016.

\section{MATERIALES Y MÉTODOS}

El diseño de este estudio es de tipo descriptivo transversal y tiene un enfoque de investigación cuantitativo. Este estudio utilizó como población 281 fichas epidemiológicas pertenecientes a los escolares de la parroquia San Sebastián, Cuenca - Ecuador. La recolección de datos fue realizada por el departamento de Investigación de la carrera de Odontología de la Universidad Católica de Cuenca en el año 2016, para esta investigación se tomaron en cuenta criterios de inclusión como: fichas de niños que tuvieron firmado el consentimiento informado y niños que cumplieron con el rango de edad determinado (12 años), mientras que para los criterios de exclusión se consideraron: fichas epidemiológicas incompletas o irregulares y niños que no poseían firmado por el representante el consentimiento informado, puesto que todos cumplían con los requerimientos no se descartó ninguna ficha y fueron usadas en su totalidad. Para analizar la frecuencia de caries se utilizó como variable independiente el índice CPOD en donde la escala de valoración es de 0.00 - 1.1 Muy bajo, 1.2 - 2.6 Bajo, 2.7 - 4.4 Medio, 4.5 - 6.5 Alto, Mayor a 6.6 Muy Alto, también se realizó una entrevista sobre la percepción del niño para determinar el "impacto al sonreír " con una escala de "si", "no", de la misma manera se examinó la variable dependiente de "severidad de la limitación al sonreír" con los niveles de "sin limitación", "poca” "regular" "mucha” en los últimos 3 meses. 


\section{REVISTA CIENTÍFICA “ESPECIALIDADES ODONTOLÓGICAS UG”. ISSN: 2600576X ÓRGANO OFICIAL DE LA FACULTAD PILOTO DE ODONTOLOGÍA DE LA UNIVERSIDAD DE GUAYAQUIL}

De esta manera se obtuvieron los datos que fueron reflejados en las fichas digitales ingresadas en el software de dominio público Epi Info, posterior a esto, la información fue exportada al programa Microsoft Excel, seguidamente se procedió a realizar la correlación de variables mediante la prueba estadística de Kendall. Para el desarrollo de este artículo científico se realizó una búsqueda exhaustiva de fuentes de información como artículos científicos no mayores a 5 años publicados en revistas de divulgación científica como: Springer, PubMed, Scielo, Latindex, UptoDate.

Este estudio no implicó ningún conflicto bioético, puesto que fue ejecutado sobre datos ya tomados de la oficina de investigación de la carrera de Odontología, de la misma manera se guardó la confidencialidad sobre los datos proporcionados.

\section{RESULTADOS}

Tabla 1. Distribución porcentual de escolares de 12 años de la parroquia San Sebastián. Distribución de escolares de la parroquia San Sebastián

\begin{tabular}{|c|c|c|}
\hline Sexo & Frecuencia & Porcentaje \\
\hline Femenino & 128 & 45.6 \\
\hline Masculino & 153 & 54.4 \\
\hline Total & 281 & 100.0 \\
\hline
\end{tabular}

En este estudio se analizaron en total 281 escolares de 12 años de la parroquia San Sebastián del cantón Cuenca, el 45,6 \% y el 54,4 \% de escolares corresponden al sexo femenino y masculino, respectivamente. Los resultados demuestran que existe un predomino del sexo masculino en relación con el sexo femenino. (Tabla 1)

Tabla 2. Análisis porcentual de los niveles de CPOD de acuerdo al sexo. Niveles de CPOD

\begin{tabular}{|c|c|c|c|}
\hline Sexo & Nivel & Frecuencia & Porcentaje \\
\hline \multirow[t]{6}{*}{ Femenino } & Muy bajo & 45 & 35.2 \\
\hline & Bajo & 19 & 14.8 \\
\hline & Medio & 30 & 23.4 \\
\hline & Alto & 19 & 14.8 \\
\hline & Muy alto & 15 & 11.7 \\
\hline & Total & 128 & 100.0 \\
\hline \multirow[t]{4}{*}{ Masculino } & Muy bajo & 62 & 40.5 \\
\hline & Bajo & 19 & 12.4 \\
\hline & Medio & 41 & 26.8 \\
\hline & Alto & 17 & 11.1 \\
\hline
\end{tabular}


REVISTA CIENTÍFICA “ESPECIALIDADES ODONTOLÓGICAS UG”. ISSN: 2600576X ÓRGANO OFICIAL DE LA FACULTAD PILOTO DE ODONTOLOGÍA DE LA UNIVERSIDAD DE GUAYAQUIL

\begin{tabular}{|c|c|c|c|}
\hline & Muy alto & 14 & 9.2 \\
\hline \multirow{2}{*}{ General } & Total & 153 & 100.0 \\
\hline \multirow{2}{*}{ Muy bajo } & 107 & 38.1 \\
\hline & Bajo & 38 & 13.5 \\
& Medio & 71 & 25.3 \\
& Alto & 36 & 12.8 \\
\hline & Muy Alto & 291 & 100.3 \\
\hline & Total & & 10.0 \\
\hline
\end{tabular}

En cuanto a los niveles de CPOD, el rango más relevante es Muy bajo con un 38,1\% en ambos sexos, seguidos por una cierta similitud en los niveles Bajo y Alto con 13,5\% y 12,8 \% respectivamente. También se observó que en los niveles Muy bajo, el sexo masculino predomina con un valor de 40,5\% en comparación del femenino con un valor de 35,2\%. (Tabla 2)

Tabla 3. Análisis porcentual sobre el impacto al sonreír. Impacto al sonreír

\begin{tabular}{|c|c|c|c|}
\hline Sexo & & Frecuencia & Porcentaje \\
\hline \multirow[t]{3}{*}{ Femenino } & No & 97 & 75.8 \\
\hline & $\mathrm{Si}$ & 31 & 24.2 \\
\hline & Total & 128 & 100.0 \\
\hline \multirow[t]{3}{*}{ Masculino } & No & 113 & 73.9 \\
\hline & $\mathrm{Si}$ & 40 & 26.1 \\
\hline & Total & 153 & 100.0 \\
\hline \multirow[t]{3}{*}{ General } & No & 210 & 74.7 \\
\hline & $\mathrm{Si}$ & 71 & 25.3 \\
\hline & Total & 281 & 100.0 \\
\hline
\end{tabular}

En lo correspondiente al impacto en el desempeño al sonreír, el 74,7\% de los escolares no mostraron limitación al sonreír, destacando ligeramente el sexo femenino con un valor del 75,8\% en comparación al sexo masculino con un 73,9\%. (Tabla 3) 


\section{REVISTA CIENTÍFICA “ESPECIALIDADES ODONTOLÓGICAS UG”. ISSN: 2600576X ÓRGANO OFICIAL DE LA FACULTAD PILOTO DE ODONTOLOGÍA DE LA UNIVERSIDAD DE GUAYAQUIL}

Tabla 4. Análisis porcentual de la severidad en la limitación al sonreír. Severidad en la limitación al sonreír

\begin{tabular}{|c|c|c|c|}
\hline Sexo & & Frecuencia & Porcentaje \\
\hline \multirow[t]{5}{*}{ Femenino } & Sin limitación & 97 & 75.8 \\
\hline & Poca limitación & 7 & 5.5 \\
\hline & Regular limitación & 12 & 9.4 \\
\hline & Mucha limitación & 12 & 9.4 \\
\hline & Total & 128 & 100.0 \\
\hline \multirow[t]{5}{*}{ Masculino } & Sin limitación & 114 & 74.5 \\
\hline & Poca limitación & 7 & 4.6 \\
\hline & Regular limitación & 18 & 11.8 \\
\hline & Mucha limitación & 14 & 9.2 \\
\hline & Total & 153 & 100.0 \\
\hline
\end{tabular}

Respecto a la severidad en la limitación al sonreír, se observó que en el apartado sin limitación el predominante fue sexo femenino con el 75,8\% a diferencia del masculino con un valor del 74,5\%, otro dato relevante que se observó fue en el apartado mucha limitación, en ambos sexos los valores son similares con un 9,4\% en el sexo femenino y 9,2\% en el masculino. (Tabla 4)

Fig. 1: Relación de los niveles de CPOD y la severidad en la limitación al sonreír.

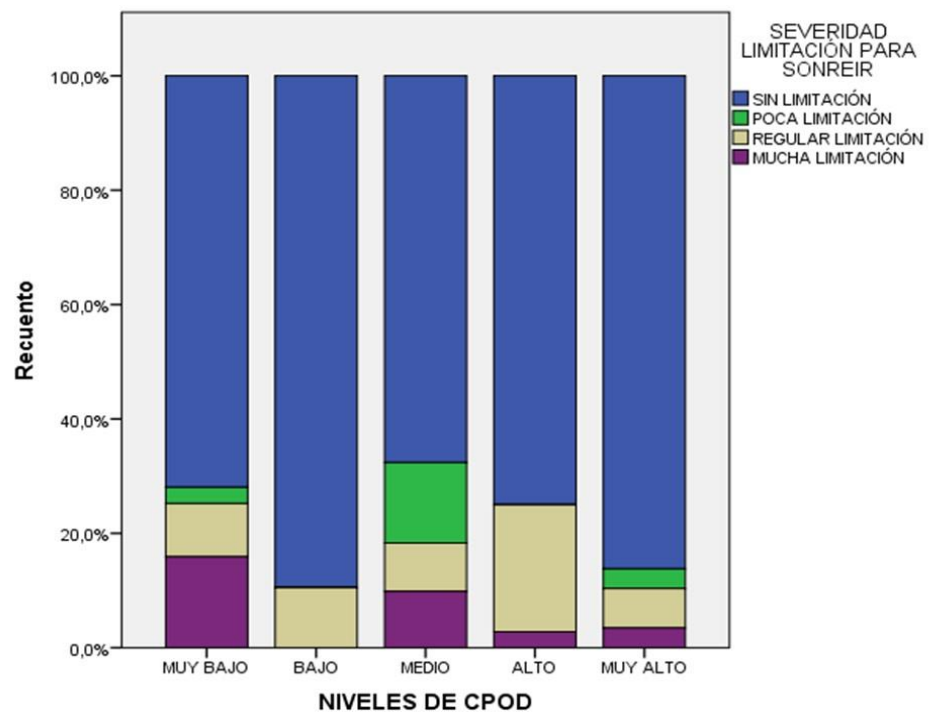

Tau b Kendall $=-0,056$

$\mathrm{P}=0,284$ 
En la figura 1 se obtuvo un Tau b Kendall $=-0,056$ y un valor $p=0,284$ lo que nos indica que existe correlación negativa inversa no significativa entre las variables nivel de CPOD y limitación al sonreír en ambos sexos.

Fig. 2: Relación de los niveles de CPOD y la severidad en la limitación al sonreír en el sexo femenino.

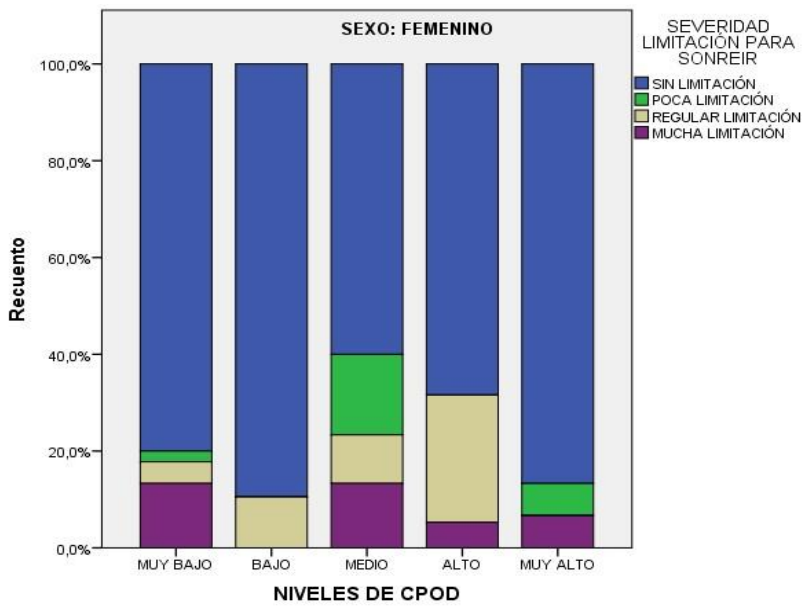

Tau b Kendall $=0,046$

$\mathrm{P}=0,554$

En la figura 2 se obtuvo un Tau b Kendall $=0,046$ y un valor $p=0,554$ lo que nos indica que existe correlación positiva directa no significativa entre las variables nivel de CPOD y limitación al sonreír en el sexo femenino.

Fig. 3: Relación de los niveles de CPOD y la severidad en la limitación al sonreír en el sexo masculino.

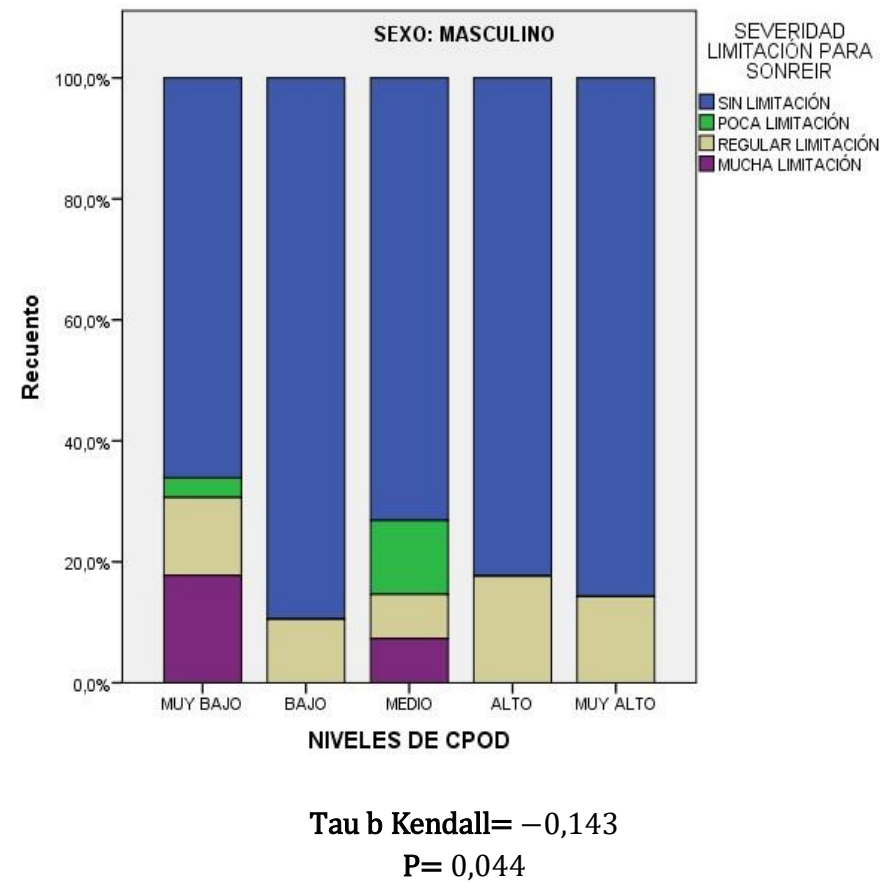

En la figura 3 se obtuvo un Tau b Kendall $=-0,143$ y un valor $p=0,044$ lo que nos indica que existe correlación positiva directa significativa entre las variables nivel de CPOD y limitación al sonreír en el sexo masculino. 


\section{REVISTA CIENTÍFICA “ESPECIALIDADES ODONTOLÓGICAS UG”. ISSN: 2600576X \\ ÓRGANO OFICIAL DE LA FACULTAD PILOTO DE ODONTOLOGÍA DE LA UNIVERSIDAD DE GUAYAQUIL}

\section{DISCUSIÓN}

La presente investigación evaluó la impresión que tiene las afecciones bucales en la calidad de vida en escolares de 12 años mediante una relación entre el índice CPOD y la limitación para sonreír en la parroquia San Sebastián, CuencaEcuador 2016, por lo tanto se determinó que en los niveles de CPOD, el rango más significativo en esta investigación es Muy bajo con un $38,1 \%$ en ambos sexos, seguidos por los niveles Bajo y Alto con 13,5\% y 12,8 \% respectivamente. También hay que destacar que en los niveles Muy bajo, el sexo masculino predomina con un valor de $40,5 \%$ en comparación del femenino, esto concuerda con Flores Magali y $\mathrm{Col}^{28}$ quienes en su estudio Prevalencia de caries dental e índice CPOD en escolares de 12 años en la parroquia baños del cantón cuenca 2016, donde evaluaron 104 escolares de 12 años de la parroquia Baños del Cantón Cuenca, siendo 44 del sexo masculino y 60 del sexo femenino, encontraron la prevalecía de un rango de severidad Muy Bajo con un porcentaje de $31.73 \%$.

Por otra parte, Córdova A, Encalada L, ${ }^{29}$ en su estudio Impacto de la caries dental en escolares de 12 años, Yanuncay, Cuenca, Ecuador, donde la muestra estuvo conformada por 132 escolares de 12 años de edad, los resultados obtenidos en ese estudio determinaron que la prevalencia de caries dental fue baja representada por el 18,2 \%, es decir, 9,1\% respectivamente para ambos sexos. Flores Magali y Col, refiere que la mayoría de niños de nuestra población presentan pocas caries. Por su parte, Vélez V, Villavicencio E, Cevallos S, Del Castillo C. ${ }^{30}$ En su estudio Impacto de la experiencia de caries en la calidad de vida relacionada a la salud bucal; Machángara, Ecuador; se evaluó una muestra de 118 escolares entre 11 y 12 años, Obtuvieron niveles Alto y Muy alto de caries con un $38 \%$ de los pacientes estudiados, por lo que esto difiere con el presente estudio.

En lo correspondiente al impacto al sonreír, el 74,7\% de los escolares no mostraron limitación al sonreír, sobresaliendo ligeramente el sexo femenino con un valor del $75,8 \%$ en comparación al sexo masculino con un 73,9\%. Esto concuerda con Córdova A, Encalada L,29 quienes en su investigación demostraron que el sexo femenino no presentó impacto al sonreír, sin embargo se evidencio afección en funciones como comer alimentos con 24,0 \% y lavarse los dientes con 21,5\%; mientras que en el sexo masculino predominó el comer alimentos con un 20,0\%; seguido de lavarse los dientes, limitación al sonreír y realizar labores con $16,4 \%$, de igual manera Orellana $L, 31$ quien en su estudio Calidad de vida relacionada a la salud bucal en escolares del ámbito Costa Sierra de ecuador participaron 166 niños entre 11 y 12 años de edad, 122 de la región Costa y 44 de la región Sierra, el $98.2 \%$ de escolares refirieron que las actividades diarias que más frecuentemente afectan la Calidad de Vida de los niños de 11 y 12 años de la provincia de Cañar son: Comer (59.0\%) y Sonreír (58.4\%); y las menos frecuentemente afectadas son: Estudiar (8.4\%) y Dormir (5.4\%).

\section{CONCLUSIONES}

A partir de los resultados obtenidos en esta investigación se concluye que el índice CPOD puede estar condicionado por el sexo en esta población, demostrando que el sexo masculino tiene un menor índice CPOD en comparación a su opuesto, esto puede deberse al tipo de alimentación y cambios hormonales en mujeres. Puesto que no presentaron gran porcentaje en la severidad de la limitación al sonreír en ambos sexos, esto evidencia que a menor índice CPOD menor limitación, en conclusión, a pesar de que existe un mínimo porcentaje de relación entre el índice CPOD y la limitación al sonreír en escolares de 12 años en la parroquia San Sebastián se pone en evidencia su existencia, también debemos considerar que el índice CPOD relativamente bajo en esta parroquia puede ser debido a que los escolares constantemente reciben capacitación de múltiples brigadas comunitarias de las diferentes instituciones públicas o privadas del cantón Cuenca, por lo tanto muchos niños tienen un conocimiento previo de cuidados y prevención de la salud bucal.

Nuestras recomendaciones para investigaciones futuras es que las entrevistas a los escolares se realicen de manera privada para evitar que sus respuestas sean influenciadas por sus compañeros, también se sugiere ampliar la población o realizar este tipo de estudios en escolares con mayor índice CPOD.

\section{REFERENCIAS BIBLIOGRÁFICAS}

1. Romo PMR, Herrera MIJ, Bribiesca GME, Rubio CJ, Hernández ZMS, Murrieta PJF. Caries dental y algunos factores sociales en escolares de Cd. Nezahualcóyotl. Bol Med Hosp Infant Mex.2015.

2. Organización Mundial de la Salud. Salud bucodental. Organización Mundial de la Salud. 2020 mar 25 [accedido 2020 jun 30];394(10194). https://www.who.int/es/newsroom/factsheets/detail/oral-health. doi:10.1016/S0140 6736(19)31146-8

3. Peres MA, Macpherson LMD, Weyant RJ, Daly B, Venturelli R, Mathur MR, Listl S, Celeste RK, Guarnizo-Herreño CC, Kearns C, et al. Oral diseases: a global public health challenge. The Lancet. 2019;394(10194):249-260. doi:10.1016/S0140-6736(19)31146-8 4. Klein H, Palmer CE. Studies on Dental Caries: VII. Sex Differences in Dental Caries Experience of Elementary School Children. Public Health Reports (1896-1970). 1938;53(38):1685. doi: $10.2307 / 4582662$

4. Graziani F, Bettini L, Petrini M. Los beneficios sistémicos de la salud periodontal: calidad de vida. Periodoncia Clínica. 2017;8(8):99-109.

5. Ayala E, Pinto G, Raza X, Herdoíza M. Plan Nacional de Salud Bucal. Quito; 2009.

6. Klein H, Palmer C, Knutson J. Estudios sobre caries dental: I. Estado dental y necesidades dentales de niños de primaria. Public Health Reports (1896-1970). 1938. 


\section{REVISTA CIENTÍFICA “ESPECIALIDADES ODONTOLÓGICAS UG”. ISSN: 2600576X ÓRGANO OFICIAL DE LA FACULTAD PILOTO DE ODONTOLOGÍA DE LA UNIVERSIDAD DE GUAYAQUIL}

7. Sánchez E, Villagrán E, Vanegas L. Estudio epidemiológico de caries dental y fluorosis. Guatemala 1999-2002. Ciudad de Guatemala; 2002.

8. Barbosa SD, Villegas F, Beltrán J. El modelo médico como generador de discapacidad. Revista Latinoamericana de Bioética. 2020;19(37-2):111-122. doi:10.18359/rlbi.4303

9. Diaz-Reissner CV, Casas-García I, Roldán-Merino J. Calidad de Vida Relacionada con Salud Oral: Impacto de Diversas Situaciones Clínicas Odontológicas y Factores SocioDemográficos. Revisión de la Literatura. International journal of odontostomatology. 2017;11(1):31-39. doi:10.4067/s0718-381x2017000100005

10. Paz C, Romo F, Schulz R. «Análisis de la percepción estética de la sonrisa» TUTOR PRINCIPAL. Santiago: Universidad de Chile; 2010.

11. Kiekens R, Kuijpers-Jagtman A, Van 't Hof M, Van 't Hof B, Maltha JC. Putative golden proportions as predictors of facial esthetics in adolescents. American Journal of

12. Orthodontics and Dentofacial Orthopedics. 2008;134(4):480-484.

Doi:10.1016/j.ajodo.2006.10.041

13. Ker AJ, Chan R, Fields H, Beck M, Rosenstiel S. Esthetics and smile characteristics from the layperson's perspective: A computer-based survey study. Journal of the

14. American Dental Association. 2008;139(10):1318-1327. doi:10.14219/jada.archive.2008.0043

15. Van P, Oosterveld P, Van G, Kuijpers-Jagtman A. Smile attractiveness: Selfperception and influence on personality. Angle Orthodontist. 2007;77(5):759-765. doi:10.2319/082606-349

16. Shimamura A, Ross J, Bennett H. Memory for facial expressions: The power of a smile.

Psychonomic

Bulletin and Review. 2006;13(2):217-222. doi:10.3758/BF03193833

17. Manjula W, Sukumar M, Kishorekumar S, Gnanashanmugam K, Mahalakshmi K. Smile: A review. Journal of Pharmacy and Bioallied Sciences. 2015;7(5):273. doi:10.4103/0975-7406.155951

18. Herrera-Salas F, Madrid-Cuautle L, Arredondo-Velázquez I. Salud oral, imagen corporal y estética bucal en adolescentes. Revista de Educación y Desarrollo. 2020;53:718.

19. Simmons RG, Rosenberg F, Rosenberg M. Disturbance in the Self-Image at Adolescence. American Sociological Review. 1973;38(5):553. doi:10.2307/2094407 19. Leal S. Oral Health Educational Intervention in At-Risk Children . San Marcos: California State University; 2020.

20. Ibarra K, Calle M, López E, Heredia D. Índice de higiene oral comunitario en escolares de 12 años. Evidencias en Odontología Clínica. 2017;3(2):46. doi:10.35306/eoc.v3i2.495

21. Bueno-Alegría JA, Gutiérrez-RojoJaime F, GuerreroCastellón MP, García-Rivera RN. Índice CPOD y ceo-d de estudiantes de una escuela primaria de la ciudad de Tepic, Nayarit. Revista Latinoamericana de Ortodoncia y Odontopediatría. 2019.

22. Abel Ferreira de Souza Junior L, Campos De Cesaro B, Antônio Barros Oliveira P, Bordin R. Dental Caries: A Schoolchildren Epidemiological profile in a Seaside Town, brazil, 2017. International Journal of Advanced
Engineering Research and Science (IJAERS). 2020;7(4):2456-1908. doi:10.22161/ijaers.74.31

23. Yen Y, Briones K, García J. Caries dental, higiene bucal y necesidades de tratamientos a beneficiarios del Proyecto Sonrisas Felices. Revista San Gregorio. 2019;0(28). doi:10.36097/RSAN.V0I28.767

24. Bulgareli J, Tanajura E, CortellazziI K, Guerra LM, Meneghim M, Ambrosano GMB, Frias A, Pereira A. Fatores que influenciam o impacto da saúde bucal nas atividades diárias de adolescentes, adultos e idosos. Revista de Saúde Pública. 2018;52:44. doi:10.11606/S15188787.2018052000042

25. Miettinen O, Lahti S, Sipilä K. Psychosocial aspects of temporomandibular disorders and oral health-related quality-of-life. Acta Odontologica Scandinavica. 2012;70(4):331-

26. 336. doi:10.3109/00016357.2011.654241

27. Borrell C, Artazcoz L. Las desigualdades de género en salud: Retos para el futuro . Rev Esp Salud Pública. 2008;82(3):245-249.

28. Ramos-Jorge J, Pordeus I, Ramos-Jorge M, Marques L, Paiva S. Impact of untreated dental caries on quality of life of preschool children: different stages and activity.

29. Community Dentistry and Oral Epidemiology. 2014;42(4):311-322. doi:10.1111/cdoe.12086

30. Flores Magali y Col. Prevalencia de caries dental e índice CPOD en escolares de 12 años en la parroquia baños del cantón cuenca 2016. Revista OACTIVA UC Cuenca.2016, 1(3).

31. Córdova A, Encalada L. Impacto de la caries dental en escolares de 12 años Yanuncay, Cuenca, Ecuador. Rev. Evid. Odontol. Clinic.2018,4(1).

32. Vélez V, Villavicencio E, Cevallos-Romero S, Del CastilloLópez C. Impacto de la experiencia de caries en la calidad de vida relacionada a la salud bucal; Machángara, Ecuador. Rev Estomatol Herediana. 2019 ,29(3).

33. Orellana L, Encalada L, Villavicencio E. Calidad de vida relacionada a la salud bucal en escolares del ámbito Costa - Sierra de Ecuador. Rev. Evid. Odontol. Clinic.2017 ,3(2).

\section{CONFLICTOS DE INTERESES}

Los autores no refieren conflictos de intereses. 